\title{
Additional value of biplane transoesophageal imaging in assessment of mitral valve prostheses
}

Kaj Groundstroem, Dylmitr Rittoo, Piotr Hoffman, Peter Bloomfield, George R Sutherland

\begin{abstract}
Objectives-To determine whether biplane transoesophageal imaging offers advantages in the evaluation of mitral prostheses when compared with standard single transverse plane imaging or the precordial approach in suspected prosthetic dysfunction.

Design-Prospective mitral valve prosthesis in situ using precordial and biplane transoesophageal ultrasonography. Setting-Tertiary cardiac referral centre. Subjects- 67 consecutive patients with suspected dysfunction of a mitral valve prosthesis (16 had bioprostheses and 51 mechanical prostheses) who underwent precordial, transverse plane, and biplane transoesophageal echocardiography. Correlative invasive confirmation from surgery or angiography, or both, was available in 44 patients.
\end{abstract}

Main outcome measures-Number, type, and site of leak according to the three means of scanning.

Results-Transverse plane transoesophageal imaging alone identified all 31 medial/lateral paravalvar leaks but only $24 / 30$ of the anterior/posterior leaks. Combining the information from both imaging planes confirmed that biplane scanning identified all paravalvar leaks. Five of the six patients with prosthetic valve endocarditis, all three with valvar thrombus or obstruction, and all three with mitral annulus rupture were diagnosed from transverse plane imaging alone. Longitudinal plane imaging alone enabled diagnosis of the remaining case of prosthetic endocarditis and a further case of subvalvar pannus formation.

Conclusions-Transverse plane transoesophageal imaging was superior to the longitudinal imaging in identifying medial and lateral lesions around the sewing ring of a mitral valve prosthesis. Longitudinal plane imaging was superior in identifying anterior and posterior lesions. Biplane imaging is therefore an important development in the study of mitral prosthesis function.

(Br Heart $\mathcal{F} 1993 ; 70: 259-265)$

Transoesophageal echocardiography has become established as an important new imaging technique in cardiology. Thousands of elective studies have been completed with a low complication rate of about $0.9 \% .^{1}$ Several reports have shown that this technique can be applied safely and is valuable in assessing mitral prosthetic valves. Transoesophageal imaging complements a precordial study and may be an essential part of a comprehensive echocardiographic examination of patients with a suspected malfunction of a mitral prosthesis. ${ }^{23}$ Transoesophageal echocardiography has the advantage of circumventing the ultrasound attenuation inherent in precordial imaging of the atrial surface of the mitral prosthesis and its sewing ring.

The initial generation of transoesophageal probes allowed imaging of the heart in only one imaging plane, the transverse axis. Recent developments in probe technology have led to the introduction of biplane transoesophageal probes. In the current generation of such probes a second transducer array producing longitudinal plane scanning is mounted proximal to the transverse plane scanner. Thus images recorded from two orthogonal planes can be displayed sequentially and by transducer tip manipulation biplane imaging can be used to produce what is in essence a more three dimensional appreciation of cardiac anatomy. ${ }^{4}$

With the addition of colour flow mapping to transoesophageal imaging, the investigation of normal and abnormal blood flows within the left atrium of patients with a mitral prosthesis has become possible. With this technique the site and extent of regurgitant jets can readily be appreciated. ${ }^{5}$ To date all transoesophageal studies of mitral valve prostheses have been restricted to imaging in the transverse plane alone using either an oesophageal or a transgastric position. With such a limitation in imaging planes it is not always possible to scan the entire valve sewing ring. ${ }^{3}$ To determine whether the addition of a second, longitudinal axis imaging plane would add important new clinical information and contribute to patient management we carried out a study comparing the information gained from either imaging plane with results from precordial ultrasonography, angiography, and surgery.

Patients and methods

The ultrasound examinations were performed at either this hospital or the Royal Infirmary, Edinburgh, between January 1991 and May 1992. Sixty seven consecutive patients with suspected mitral prosthesis dysfunction were 
Table 1 Clinical data on 67 consecutive patients with clinically suspected mitral prosthetic dysfunction

\begin{tabular}{ll}
\hline Time since implantation: & \\
Mean (SD) (years) & $5 \cdot 5(5 \cdot 2)$ \\
Range & 1 month-16 years \\
Age (years): & $64(10)$ \\
Mean (SD) & $35-80$ \\
Range & 51 \\
Prosthesis type (No of patients): & 16 \\
Mechanical & 11 \\
Tissue valve & \\
Concomitant aortic valve &
\end{tabular}

studied initially by precordial echocardiography and subsequently by biplane transoesophageal echocardiography. All the studies were recorded on continuous videotape and were subsequently analysed and interpreted by two independent observers. For each study the transverse plane images were analysed first with subsequent analysis of the longitudinal plane images being carried out. The information derived from each imaging plane study was then compared and contrasted. Any diagnostic disagreements (there were none) were to be settled by reaching a consensus decision after a further joint review of the videotape. Table 1 shows the patients' demographic and clinical details.

\section{TRANSOESOPHAGEAL ECHOCARDIOGRAPHY}

Two transoesophageal echocardiographic imaging systems were used: an Acuson 5.6 $\mathrm{MHz} 64$ channel biplane probe (attached to Acuson $128 / \mathrm{XP10}$ ) or an ATL $4.8 \mathrm{MHz} 48$ channel biplane probe (attached to ATL Ultramark 9). Both probes have integrated colour flow mapping and pulsed Doppler capabilities, and, in addition, the Acuson
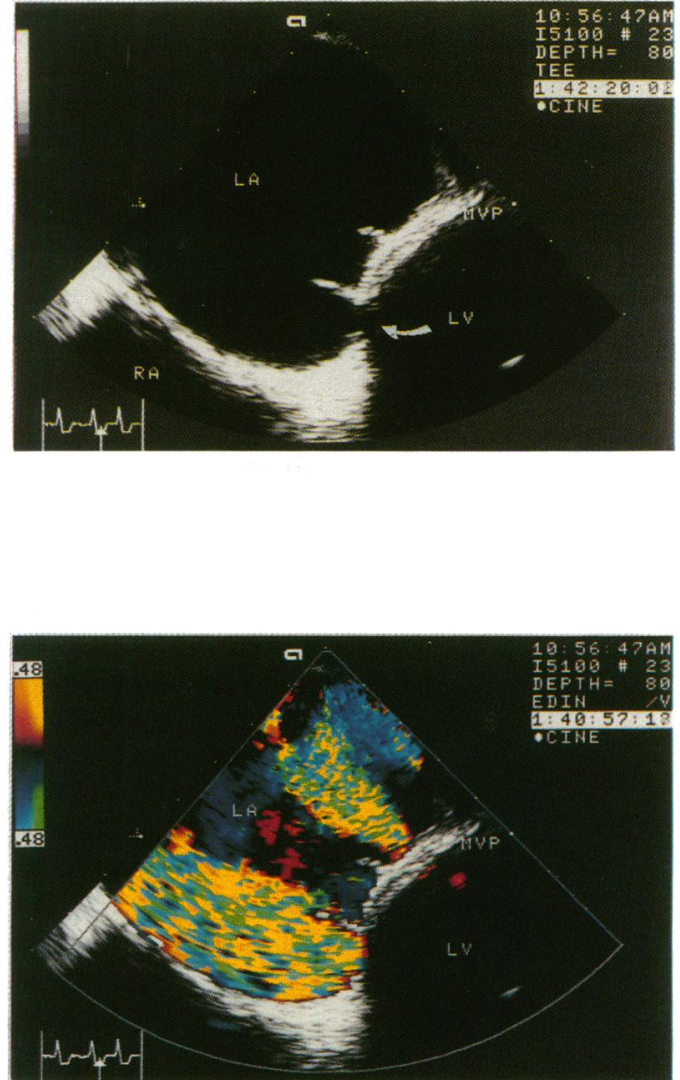

Figure 1 Transoesophageal transverse plane image of severe regurgitation in patient with mechanical mitral prosthesis. Top: Single medial site of dehiscence (arrow), the only abnormality found in this plane. Bottom: Corresponding colour flow map showing two significant pathological paravalvar regurgitant jets. The greater leak is through the medial dehiscence. The origin of the second paravalvar leak could not be assessed in this plane but was shown to be sited posteriorly by longitudinal plane scanning. ( $L A=$ left atrium, $L V=$ left ventricle, $R A=$ right atrium, $M V P=$ mitral valve prosthesis.)
Figure 2

Transoesophageal transverse plane image of lateral dehiscence (arrow) of a mechanical mitral prosthesis (left). Colour flow mapping shows gross systolic (top right) and diastolic (lower right) paravalvar flow through area of dehiscence. Site of origin and severity of the paravalvar leakage were best displayed in this plane. ( $L A=$ left atrium, $L V=$ left ventricle $M V P=$ mitral valve prosthesis, LUPV = left upper pulmonary vein. $L A A=$ left atrial appendage, $A D=$ aortic valve.)

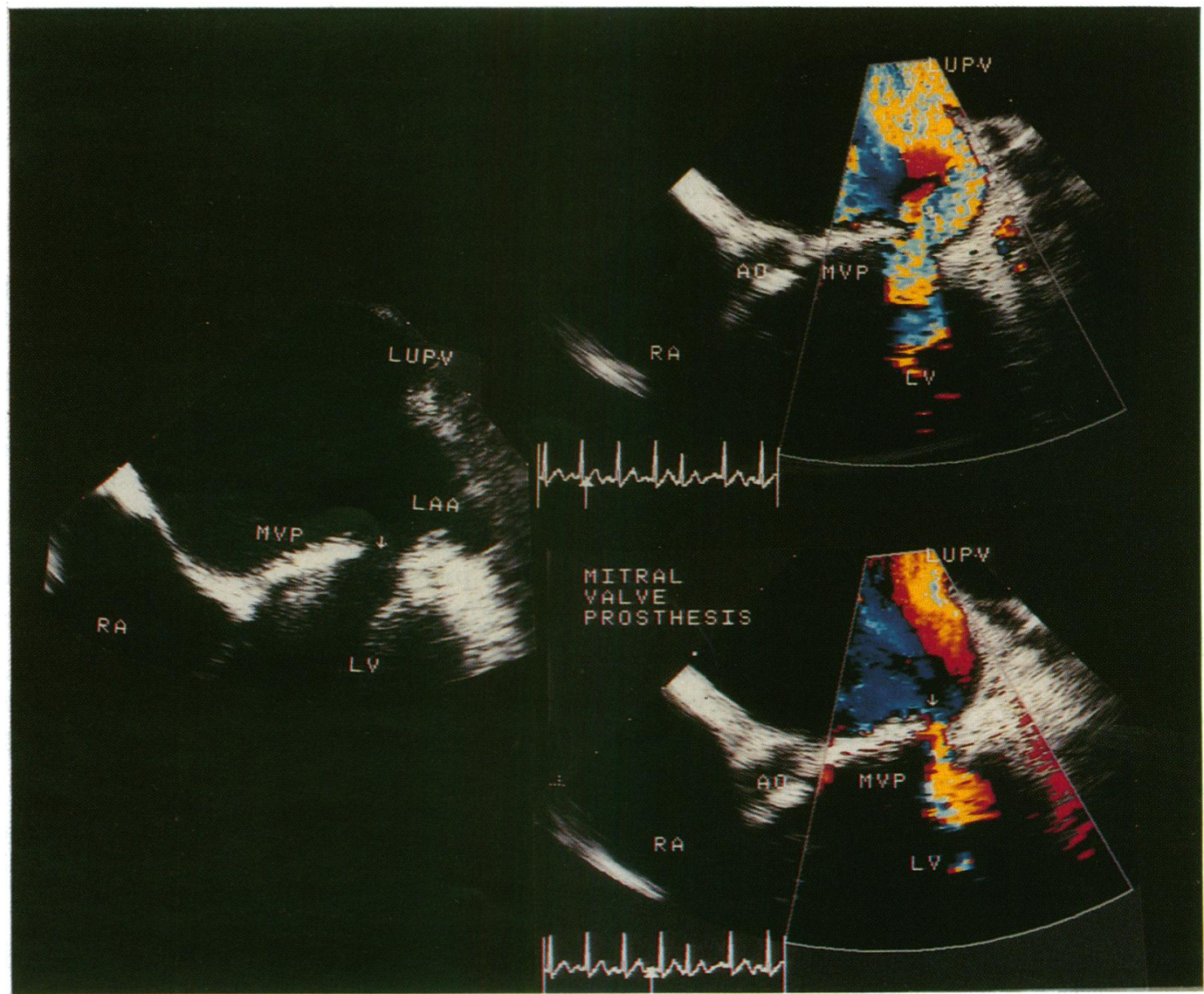




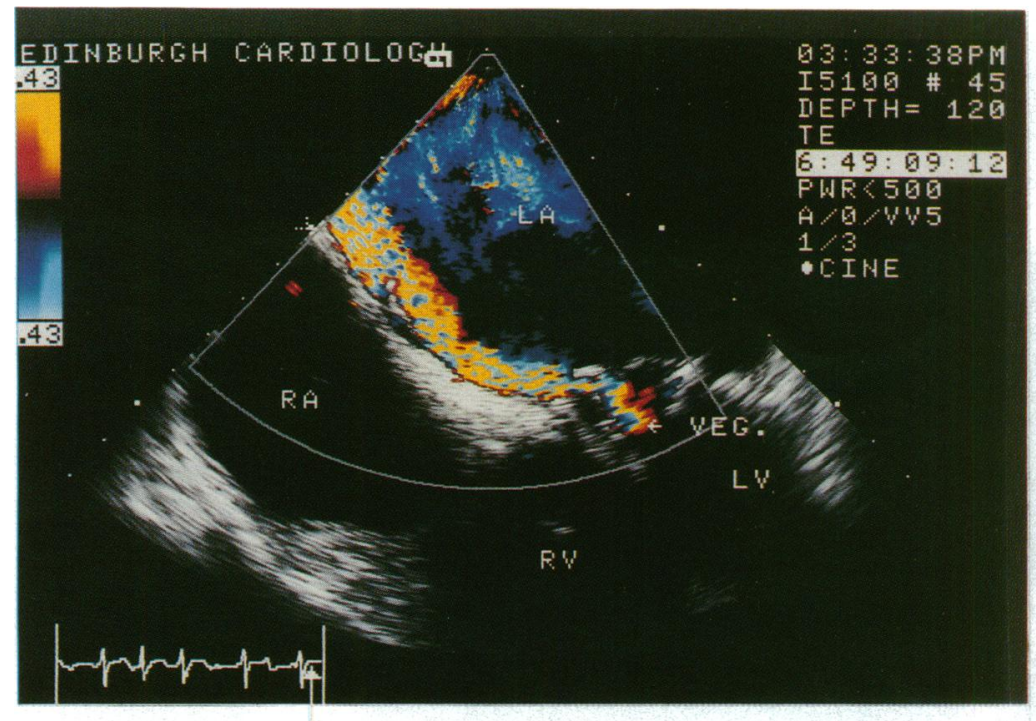

Figure 3 Transoesophageal transverse plane image of central orifice regurgitation in a mitral bioprosthesis in a patient with infective endocarditis affecting septal leaflet of valve. The causative vegetation is indicated with an arrow. There were no paravalvar leaks in this case. ( $L A=$ left atrium, $L V=$ left ventricle, $R A=$ right atrium, $R V=$ right ventricle, $V E G=$ vegetation.)

probe has an integrated continuous wave Doppler capability.

The patients all abstained from oral intake for at least four hours before the study. A history was taken to exclude possible oesophageal disease and difficulties in swallowing. An intravenous cannula was routinely inserted in a peripheral vein. The pharynx was sprayed with an aerosol of lignocaine. All patients were sedated with $2 \cdot 5-5 \mathrm{mg}$ intravenous midazolam 5-10 minutes before introduction of the probe. The examinations were then performed with the patient lying in the left lateral decubitus position. During the study additional midazolam was administered when considered necessary to avoid patient discomfort. Patients under 40 years of age in whom excess salivation was more likely were given $0.2 \mathrm{mg}$ glycopyrrolate intravenously to reduce secretions. No antibiotics for prophylaxis against endocarditis were given, which is our routine practice.

The examination of the mitral valve prosthesis was started by scanning the prosthesis ring and the left atrium in the transverse plane using standard views of four and five chambers of the heart. Instrument settings such as colour gain, depth, transmit power, and colour flow map were adjusted individually to obtain both the best quality cross sectional images and the maximum jet area for any mitral regurgitation. During the examination the vertical position of the probe was finely adjusted to visualise the different parts of the prosthesis and left atrium. After the optimal imaging position had been deter-

Table 2 Results of single plane and cross sectional imaging of valvar and paravalvar regurgitation leaks in 47 patients with suspected mitral prosthetic dysfunction

\begin{tabular}{llll}
\hline Site of leak & Transverse plane & Longitudinal plane & Both \\
\hline Anterior/posterior paravalvar $(\mathrm{n}=30)$ & 24 & 30 & 30 \\
Medial/lateral paravalvar $(\mathrm{n}=31)$ & 31 & 24 & 31 \\
Central orifice $(\mathrm{n}=10)$ & 10 & 10 & 10 \\
Total $(\%)$ & $65(92)$ & $64(90)$ & $71(100)$ \\
\hline
\end{tabular}

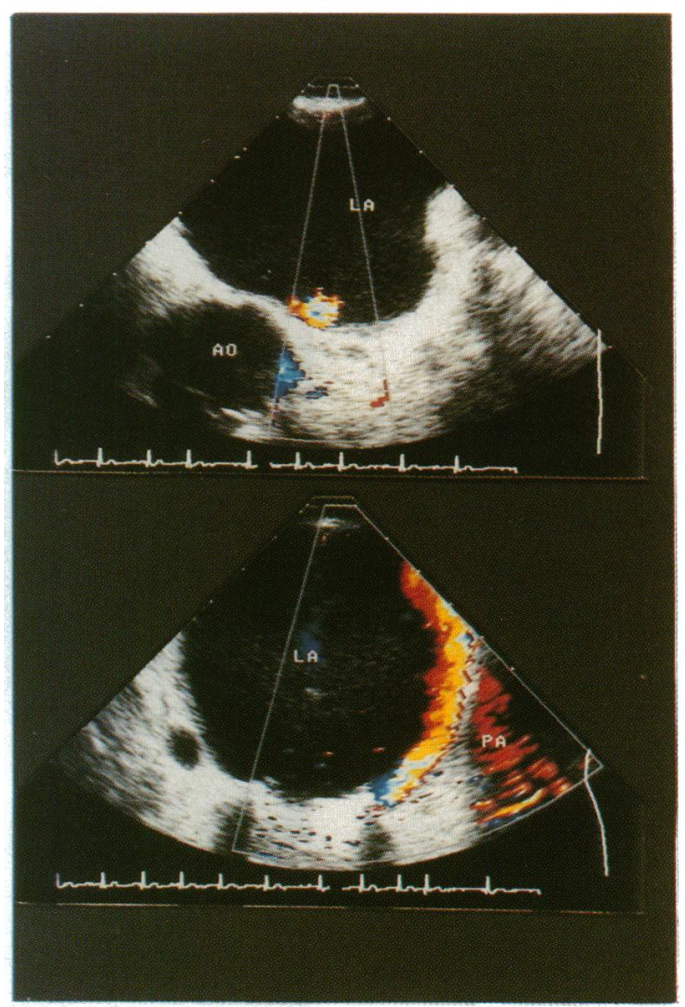

Figure 4 Biplane transoesophageal echocardiographic imaging of anterior paravalvar leak in a mechanical mitral valve prosthesis. These images show the difficulty in visualising anterior paravalvar leaks with transverse plane scanning alone. Top: Transverse plane image showing anterior paravalvar regurgitation jet in cross section as it passes through the anteriorly directed interrogating plane. The course of the regurgitant jet is such that complete assessment of its size and direction is impossible in this plane alone. Bottom: Longitudinal plane image showing the jet's width and extension as it flows along the anterior left atrial wall. ( $L A=$ left atrium, $A O=$ ascending aorta, $P A=$ pulmonary artery.)

mined, visualisation of anterior-superior and posterior-inferior parts of the prothesis and sewing ring was completed by anteflexing and retroflexing the flexible tip of the transducer. Fine adjustments of the imaging plane were accomplished by lateral or medial flexion of the tip of the probe.

Subsequent scanning in the longitudinal plane was performed from the same position as the transverse imaging. Gradual rotation of the probe was performed to scan different longitudinal planes, with emphasis being placed on the visualisation of the lateral and medial margins of the valve sewing ring. Anteflexion and retroflexion of the probe together with lateral and medial flexion were used for fine adjustments of the imaging plane. Transgastric scanning of the mitral prosthesis in both imaging planes was attempted in every case by advancing the probe into the stomach and anteflexing its tip.

CLASSIFICATION OF MITRAL REGURGITATION Each type of mechanical valvar prosthesis has an inherent characteristic leakage pattern specific to the valve type. ${ }^{36}$ In any study of prosthetic or paraprosthetic mitral leakage this inherent leakage pattern has to be distinguished from pathological mitral regurgitation. The severity of pathological mitral 
Figure 5 Biplane transoesophageal imaging of multiple paravalvar mechanical mitral prosthesis. A: Transverse suspected anterior dehiscence (arrow). B: Longitudinal plane image more clearly showing single area of anterior prosthesis dehiscence (arrow). C: Colour flow mapping in showing two pathological paravalvar mitral regurgitant jets. The origin regurgitant jet is visible, but its extension cannot be lateral regurgitant jet whose origin cannot be assessed in this plane. D: Colour Doppler flow mapping in the

longitudinal plane showing that both regurgitant jets are extensive. One is sited anteromedially, the other, larger jet is sited posterolaterally. ( $L A=$ left atrium, $L V=$ left ventricle, $R A=$ right atrium.) leaks in a patient with a plane image showing site of the transverse plane of the anterior paravalvar appreciated. There is also a

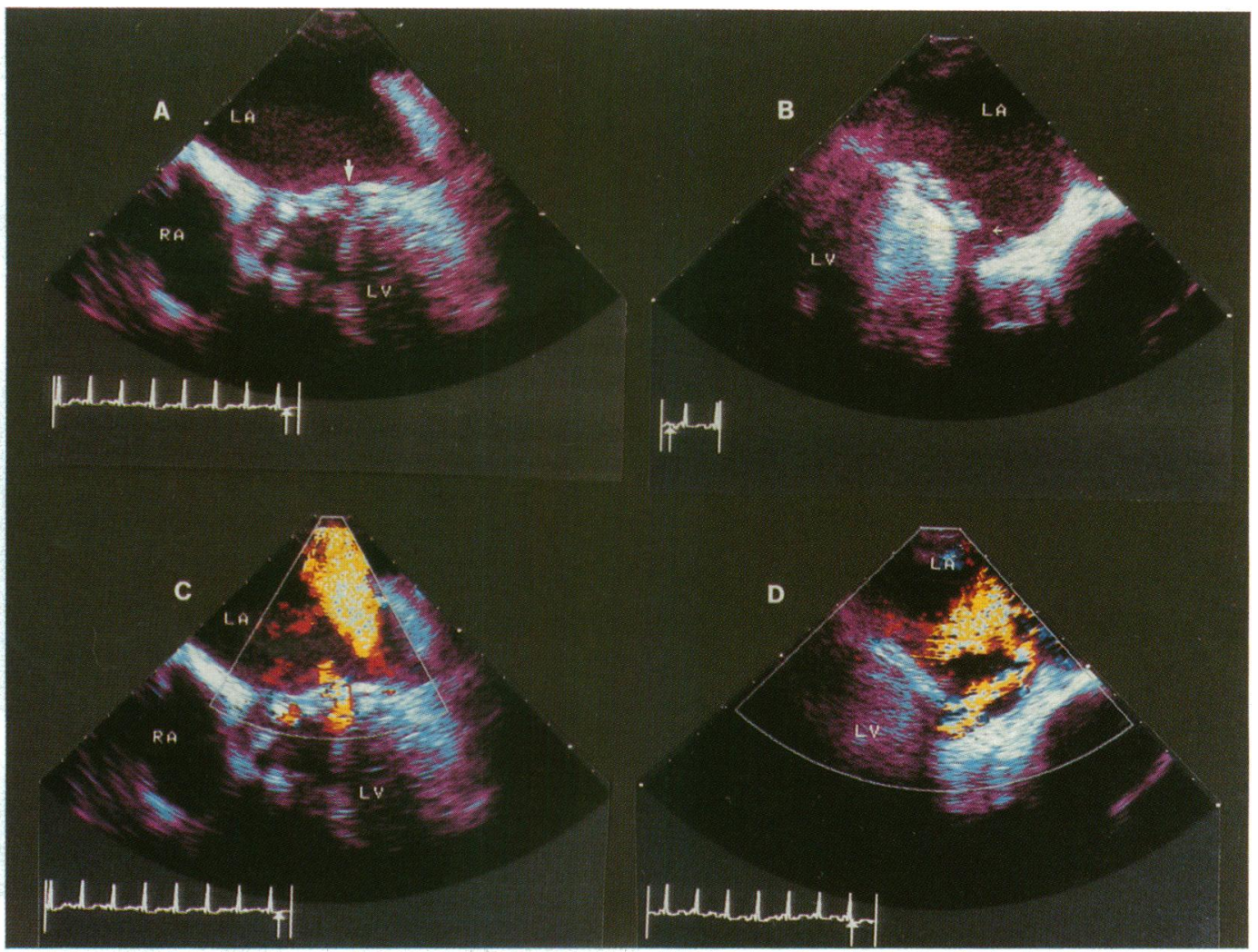

regurgitation was based on evaluating the onset, duration, length, width, and colour of the regurgitant flow. ${ }^{7}$ A regurgitant jet observed either during part or whole of systole of less than $30 \mathrm{~mm}$ in length and less than $10 \mathrm{~mm}$ in width and predominantly red in colour was classified as a marker of mild regurgitation. A multicoloured holosystolic jet less than $30 \mathrm{~mm}$ long and less than $10 \mathrm{~mm}$ wide that did not reach into the pulmonary veins was considered to be associated with moderate regurgitation. Colour jets that fulfilled the criteria of at least moderate regurgitation and extended into the pulmonary veins were considered to be markers of severe regurgitation.

\section{CORRELATIVE CONFIRMATION}

The echocardiographic findings were confirmed by surgery in 19 patients and by catheterisation in 25 patients. After complete precordial and transoesophageal echocardiographic examinations 15 of the remaining patients were classified as having normal ultrasound findings and eight as having trivial valvar or paravalvar leaks. These findings were considered to be diagnostic and to
Table 3 Results of single plane and cross sectional imaging in 13 patients with concomitant mitral prosthetic disease

\begin{tabular}{lccc}
\hline Lesion & Transverse plane & Longitudinal plane & Both \\
\hline Endocarditis $(\mathrm{n}=6)$ & 5 & 6 & 6 \\
Thrombus/obstruction $(\mathrm{n}=3)$ & 3 & 2 & 3 \\
Annulus rupture $(\mathrm{n}=3)$ & 3 & 2 & 3 \\
Subvalve pannus $(\mathrm{n}=1)$ & 0 & 1 & 1 \\
Total $(\%)$ & $11(85)$ & $11(85)$ & $13(100)$ \\
\hline
\end{tabular}

proceed to further invasive studies would have subjected the patients to a small, but unnecessary risk of complications. Thus no correlative angiographic or surgical information is available in these 23 cases.

\section{Results}

TRANSVERSE AND CROSS SECTIONAL IMAGING OF MITRAL PROSTHETIC AND PARAPROSTHETIC REGURGITATION

The tranverse plane was able to visualise the complete medial and lateral portions of the mitral annulus in every case. The origin and extension of all pathological regurgitant jets in these areas (figs 1 and 2) and within the central orifice (fig 3 ) of the prosthesis could be accurately assessed in every case. In the case of multiple regurgitant jets in which the origin of some jets was anteriorly or posteriorly around the sewing ring, the origin (fig 1). This transverse plane image shows a medial regurgitation jet passing through the area of medial dehiscence but the origin and true extension of the second (posterior) jet could not be assessed by transverse plane scanning despite a careful imaging study using anteflexion and retroflexion of the rior aspects of the valve sewing ring. Eccentric regurgitant jets may have a site of origin and course that requires biplane imaging for complete assessment (fig 4).

During the transoesophageal echocardiographic studies the inherent patterns of mitral regurgitation that are characteristic for each valve type could be visualised for most could not be assessed by imaging in this plane probe tip to visualise the anterior and poste- 


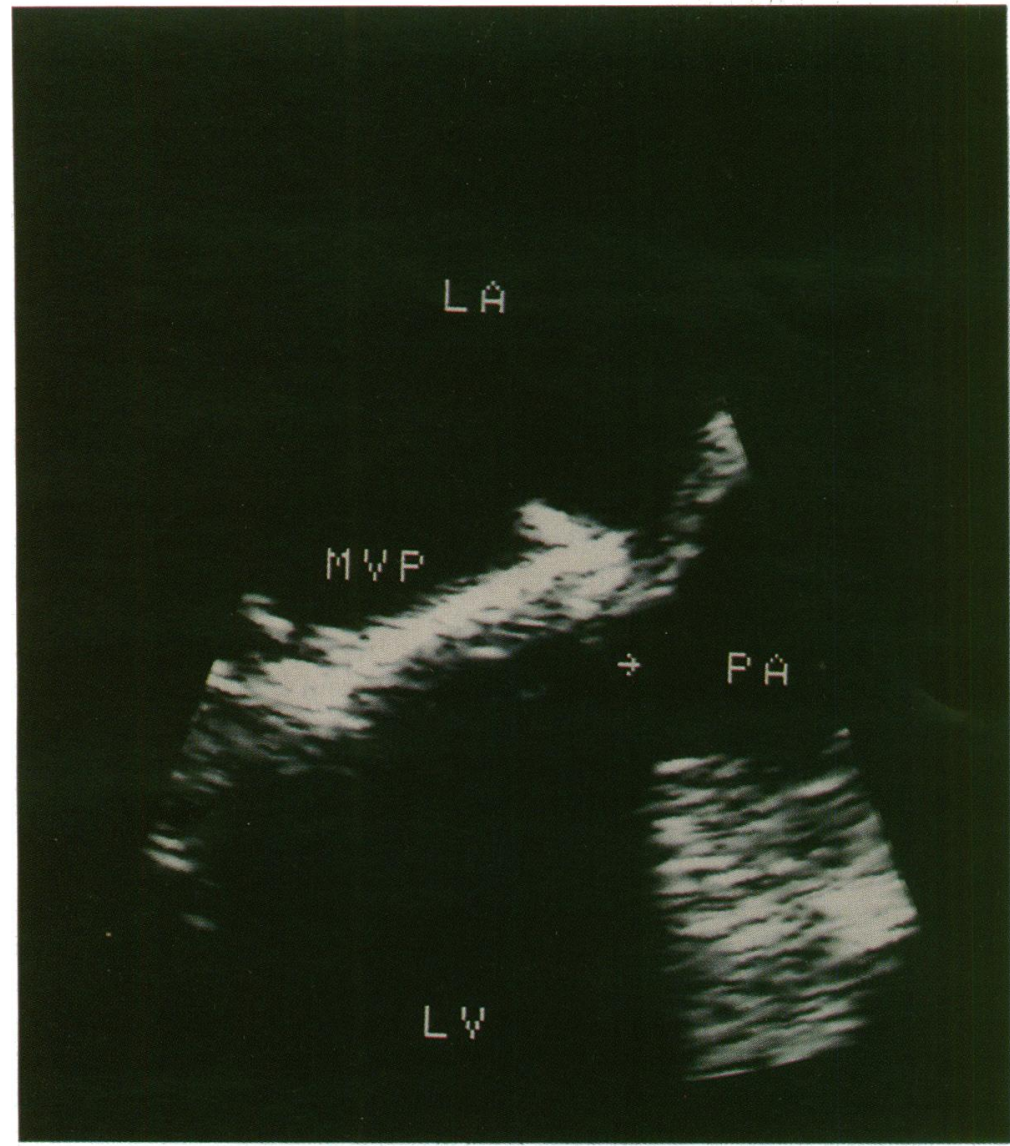

Figure 6 Biplane transoesophageal echocardiographic image of a subannular rupture and pseudoaneurysm in a patient with a mechanical mitral prosthesis. Top: Transverse plane image showing the pseudoaneurysm (arrow) below the lateral part of the annulus. Bottom: Longitudinal plane image showing that the pseudoaneurysm (arrow) extends to the anterior part of the annulus. ( $L A=$ left atrium, $L V=$ left ventricle, $M V P=$ mitral valve prosthesis, $L A A=$ left atrial appendage, $P A=$ pseudoaneurysm.)

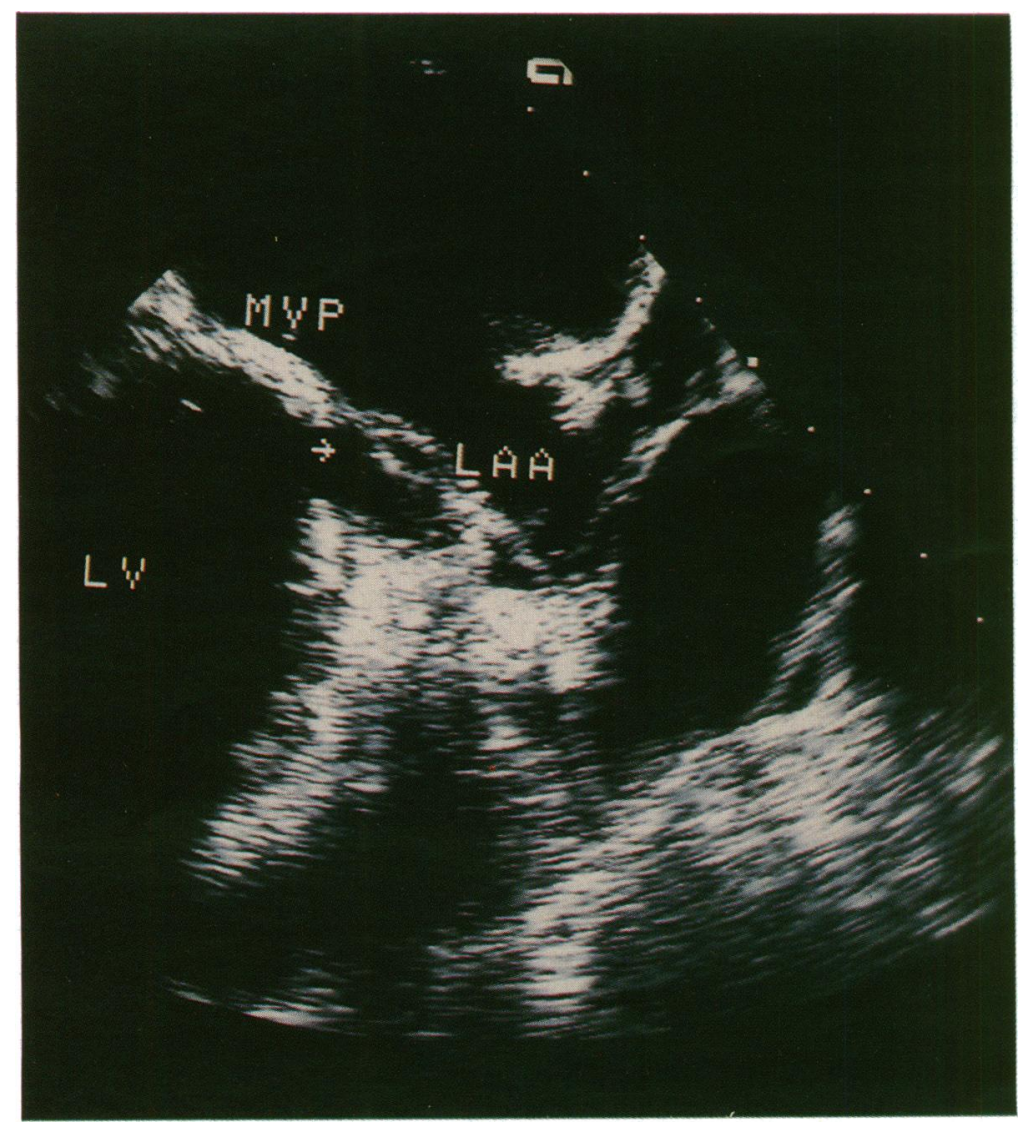

mechanical prostheses using either imaging plane. Pathological mitral regurgitation was present in 39 of the 67 patients as their only pathological lesion. In 18 patients there was a single paravalvar leak, in eight patients two paravalvar leaks were present, in four patients three paravalvar leaks, in four patients one central leak, and in five patients the combination of a central and one or more paravalvar leaks. Eight patients had mitral regurgitation as a direct consequence of concomitant prosthetic valve disease. With transverse plane scanning alone it was difficult in some cases to determine the origin of the regurgitant jet as being either central orifice or paraprosthetic. This was particularly true of the patients with concomitant central and paraprosthetic leaks in whom the correct three dimensional appreciation of the origin sites of the multiple regurgitant jets required detailed biplane assessment. Table 2 shows the comparison between transverse plane and biplane transoesophageal imaging for assessing the site and number of all valvar and paravalvar leaks.

TRANSVERSE PLANE VERSUS BIPLANE

TRANSOESPHAGEAL IMAGING OF MITRAL

PROSTHESIS DISEASE

Biplane echocardiography was superior to single transverse plane scanning in both localising and measuring pathological changes within and around the valve. In a case of severe paraprosthetic leakage due to two areas of dehiscence of the sewing ring two regurgitant jets were visible with the transverse plane. To localise their extension and both sites of dehiscence, however, scanning in two orthogonal planes was needed. Biplane imaging showed that both regurgitant jets were of similar size and that the patient had two large paravalvar leaks (fig 5).

Depending on their localisation, vegetations were often seen in both imaging planes (fig 3). Only biplane scanning was able to detect all of them. In two cases of mitral annulus rupture and pseudoaneurysm formation, one was not detected by longitudinal plane scanning. In this case, however, the lateral rupture site and the associated pseudoaneurysm was small. The other case of mitral annulus rupture was readily detected in both planes, but its posterolateral extension could not be appreciated without information from both planes (fig 6). Table 3 shows diagnostic accuracy of transverse plane and biplane scanning in assessing mitral prosthetic disease.

\section{Discussion}

COLOUR DOPPLER FLOW ASSESSMENT OF MITRAL REGURGITATION BY TRANSVERSE PLANE AND BIPLANE TRANSOESOPHAGEAI ECHOCARDIOGRAPHY

Omoto et al showed in their biplane assessment of colour flow Doppler mitral regurgitant jets that the transverse and longitudinal plane jet areas can differ by more than half in the same patients. ${ }^{5}$ The best correlation with 
contrast angiography was achieved when jet areas from both planes were combined. In their assessment of mitral regurgitation by biplane transoesophageal echocardiography Yoshida et al found that the best correlation with angiography was obtained when the greater measurement from two different planes was considered. ${ }^{8}$ Thus, both studies were able to show that in comparison with conventional transverse echocardiography, mitral regurgitant jet areas from biplane imaging correlate better with contrast angiography. Therefore, instead of measuring mitral regurgitation jets by planimetry we investigated whether adding longitudinal plane imaging to the transoesophageal echocardiographic examination could give additional new information about the origin, number, and extension of mitral regurgitant jets. Furthermore, prosthetic paravalvar regurgitation jets are often crescent shaped and adhere to the atrial wall, making their planimetric assessment less reliable. Our observers agreed in all cases on the extent, number, and severity of the regurgitant jets seen in either imaging plane. This may be because the studies were subsequently reviewed in slow motion from videotapes and because biplane approach gives a second image of regurgitant jets, thus making visual assessment more confident.

We found transoesophageal biplane colour Doppler flow mapping of regurgitant jets more reliable than conventional transverse plane assessment. A proportion of regurgitant jets situated around the anterior and posterior aspects of the annulus were not visualised by imaging in the transverse plane. The size and direction of a jet which may be eccentric and which may move out of the imaging plane might be appreciated better by biplane scanning in the same way that jet morphology in native mitral regurgitation is better evaluated by scanning multiple planes from the transthoracic approach. ${ }^{9}$ Complete spatial localisation of many of the regurgitant jets was not feasible using only the transverse plane. Superimposition of a paraprosthetic leak on a minor prosthetic leak, due to the inherent properties of the prosthesis, may on single plane scanning create the impression of a single broad based paraprosthetic regurgitation jet. Jets that may seem narrow in one plane can in the other plane be broad and occupy a major portion of the left atrial area. It is known that regurgitant blood flows along the atrial wall may become artificially narrow in one plane because of the Coanda effect. ${ }^{10}$ In our experience, biplane imaging can consistently allow an accurate spatial evaluation of jet morphology.

\section{ASSESSMENT OF VALVAR AND PARAVALVAR STRUCTURES BY TRANSVERSE PLANE AND BIPLANE TRANSOESOPHAGEAL ECHOCARDIOGRAPHY}

Biplane imaging from the oesophagus will normally allow a complete scan of valve sewing ring. An alternative approach to visualisation of the whole prosthetic sewing ring is to use transgastric imaging. To carry out transgastric scanning the probe is advanced into the stomach and the tip anteflexed to obtain long and short axis views of both the sewing ring and the left ventricle. This approach offers the opportunity of localising vegetations and regurgitant blood flows around the sewing ring. Even with the transgastric approach, however, mechanical prostheses can cast a wide acoustic shadow on their atrial aspect, again making this region difficult to inspect. With the help of longitudinal plane imaging from the transgastric position the acoustic shadow may be minimised and better imaging of the atrial surface of the valve and its adjacent structures obtained.

We found that detection of mitral annulus rupture was best achieved by transverse plane scanning when the lesion was extensive. Smaller areas of rupture under the prosthesis, including the exact localisation and the extent of associated sewing ring dehiscence, could only be appreciated by integrating the information from both planes. Thrombotic material and vegetations on and around the valve were shown in all cases with a biplane approach whereas scanning only in the transverse plane failed to recognise both anterior and posterior lesions that were of less than moderate size. In the patient with subvalve pannus only longitudinal plane scanning was able to identify this lesion. As noted earlier, however, the obstructed transvalvar flow and the nature of the subvalve obstruction was best defined during precordial scanning.

\section{LIMITATIONS OF THE STUDY}

The evaluation of any new diagnostic technique is dependent on a comparative gold standard. In our patients cineangiography or surgical confirmation was available in only 25 and 19 patients respectively. In the patients with surgical results the correlation was excellent between findings on cross sectional transoesophageal echocardiography and the subsequent surgical description of mitral prosthetic disease. Multiplane cineangiography cannot act as the gold standard as it cannot define the number or site of multiple regurgitant jets with sufficient accuracy; this is only possible using biplane transoesophageal echocardiography (see above). Therefore, biplane transoesophageal echocardiography was accepted as the gold standard for assessing the number and site of regurgitant jets. Twenty three patients in this series had either normal physiological valve leakage or trivial pathological valvar or paravalvar leakage at transoesophageal echocardiographic study. Transthoracic colour and spectral Doppler echocardiography did not suggest valvar obstruction. Therefore, for these patients, on the basis of clinical, echocardiographical, and bacteriological, information, we decided that no subsequent invasive examinations were necessary to confirm the transoesophageal findings. A clinically important paravalvar leak may have been missed, but clinical examination, 
precordial Doppler findings, and the cross sectional transoesophageal echocardiographic study were all in accordance with trivial or no valve abnormality. Therefore we doubt that significant disease was missed in this patient group. In the patients with cineangiography correlation on the severity of mitral regurgitation was good but angiography consistently failed to detect the number and site of jets.

\section{CONCLUSIONS}

As in earlier comparative studies on the transoesophageal assessment of mitral prosthetic function, the information that could be yielded from the transoesophageal window was far superior to that offered by precordial scanning. We found however, that the further addition of longitudinal plane scanning to the transoesophageal examination can allow a much improved definition of the anterior and posterior aspects of the mitral annulus and sewing ring when compared with single transverse plane scanning. Transverse plane imaging remained superior to longitudinal imaging in detecting medial or lateral valve or paravalvar lesions. Also during colour flow mapping studies the integrated biplane approach yielded more information than single transverse plane studies on the number, width, length, and origin of regurgitant jets, thus offering more accurate information and providing a higher degree of diagnostic confidence. In addition, small-that is, less than $3 \mathrm{~mm}$ - but clinically relevant lesions such as small vegetations or thrombi were more easily diagnosed by scanning in two orthogonal planes than in one. We therefore conclude that biplane transoesophageal imaging is a significant improvement over single transverse plane imaging in evaluating mitral prosthetic function.

KG is supported by the Finnish Cardiac Society and PH by the European Society of Cardiology. GRS is a senior research fellow of the British Heart Foundation.

1 Daniel WG, Erbel R, Kasper W, et al. Safety of Transoesophageal echocardiography-a multicenter survey of 10,419 examinations. Circulation 1991;83:817-21.

2 Alam M, Rosman H, Polanco GA, Sheth M, Garcia R Serwin JB. Transesophageal echocardiographic features of stenotic bioprosthetic valves in the mitral and tricuspid valve positions. Am $\mathcal{F}$ Cardiol 1991;68:689-90.

3 Khanderia BK, Seward JB, Oh JK, et al. Value and limitations of transesophageal echocardiography in assessmen of mitral valve prostheses. Circulation 1991;83:1956-68.

4 Stümper O, Fraser AG, Ho SY, et al. Transoesophageal echocardiography in the longitudinal axis. Correlation between anatomy and images a

5 Omoto R, Kyo S, Matsumura M, et al. Evaluation of biplane colour Doppler transesophageal echocardiogra-
bito phy in 200 consecutive patients. Circulation 1992;85 $1237-47$.

6 Nihoyannopoulos P, Kambouroglou D, Athanassopoulos G, Nadazdin A, Smith P, Oakley CM. Doppler haemodynamic profiles of clinically and echocardiographically normal mitral and aortic valve prostheses. Eur Heart $f$ 1992;13:348-55.

7 Taams MA, Gussenhoven EJ, Cahalan MK, et al. Transesophageal Doppler colour flow imaging in the detection of native and Björk-Shiley mitral valve regurgitation. 7 Am Coll Cardiol 1989;13:95-9.

8 Yoshida K, Yoshikawa J, Hozumi T, Akasaka T, Fukaya $T$. Assessment of mitral regurgitation by biplane T. Assessment of mitral regurgitation by biplane
transesophageal colour mapping. Circulation 1990;82: transesoph

9 Smyllie JH, Sutherland GR, Geuskens R, Dawkins K, Conway N, Roelandt JR. Doppler colour flow mapping in the diagnosis of ventricular septal rupture and acute mitral regurgitation after myocardial infarction. $\mathcal{F} A m$ Coll Cardiol 1990;15:1449-55.

10 Chao K, Moises VA, Shandas R, Elkadi T, Sahn DJ, Weintraub R. Influence of the Coanda effect on colour Doppler jet area and colour encoding: in vitro studies using colour Doppler flow mapping. Circulation 1992 85:333-41. 